\title{
Martingale representation for degenerate diffusions
}

\author{
A.S. Üstünel \\ Bilkent University, Math. Dept., Ankara, Turkey
}

\section{A R T I C L E I N F O}

\section{Article history:}

Received 10 May 2018

Accepted 14 December 2018

Available online 17 December 2018

Communicated by L. Gross

\section{Keywords:}

Entropy

Degenerate diffusions

Martingale representation

Relative entropy

Innovation process

Causal Monge-Ampère equation
A B S T R A C T

Let $(W, H, \mu)$ be the classical Wiener space on $\mathbb{R}^{d}$. Assume that $X=\left(X_{t}\right)$ is a diffusion process satisfying the stochastic differential equation $d X_{t}=\sigma(t, X) d B_{t}+b(t, X) d t$, where $\sigma:[0,1] \times C\left([0,1], \mathbb{R}^{n}\right) \rightarrow \mathbb{R}^{n} \otimes \mathbb{R}^{d}, b:[0,1] \times C\left([0,1], \mathbb{R}^{n}\right) \rightarrow$ $\mathbb{R}^{n}, B$ is an $\mathbb{R}^{d}$-valued Brownian motion. We suppose that the weak uniqueness of this equation holds for any initial condition. We prove that any square integrable martingale $M$ w.r.t. to the filtration $\left(\mathcal{F}_{t}(X), t \in[0,1]\right)$ can be represented as

$$
M_{t}=E\left[M_{0}\right]+\int_{0}^{t} P_{s}(X) \alpha_{s}(X) \cdot d B_{s}
$$

where $\alpha(X)$ is an $\mathbb{R}^{d}$-valued process adapted to $\left(\mathcal{F}_{t}(X), t \in\right.$ $[0,1])$, satisfying $E \int_{0}^{t}\left(a\left(X_{s}\right) \alpha_{s}(X), \alpha_{s}(X)\right) d s<\infty, a=\sigma^{\star} \sigma$ and $P_{s}(X)$ denotes a measurable version of the orthogonal projection from $\mathbb{R}^{d}$ to $\sigma\left(X_{s}\right)^{\star}\left(\mathbb{R}^{n}\right)$. In particular, for any $h \in H$, we have

$$
\begin{aligned}
E\left[\rho(\delta h) \mid \mathcal{F}_{1}(X)\right]= & \exp \left(\int_{0}^{1}\left(P_{s}(X) \dot{h}_{s}, d B_{s}\right)\right. \\
& \left.-\frac{1}{2} \int_{0}^{1}\left|P_{s}(X) \dot{h}_{s}\right|^{2} d s\right),
\end{aligned}
$$

where $\rho(\delta h)=\exp \left(\int_{0}^{1}\left(\dot{h}_{s}, d B_{s}\right)-\frac{1}{2}|H|_{H}^{2}\right)$. In the case the process $X$ is adapted to the Brownian filtration, this

E-mail address: ustunel@fen.bilkent.edu.tr. 
result gives a new development as an infinite series of the $L^{2}$-functionals of the degenerate diffusions. We also give an adequate notion of "innovation process" associated to a degenerate diffusion which corresponds to the strong solution when the Brownian motion is replaced by an adapted perturbation of identity. This latter result gives the solution of the causal Monge-Ampère equation.

() 2018 Elsevier Inc. All rights reserved.

\section{Introduction}

The representation of random variables with the stochastic integrals with respect to some basic processes has a long history and also it has very important applications, e.g., in signal theory, filtering, optimal control, finance, stochastic differential equations, in physics, etc. Let us recall the question: assume that we are given a certain semimartingale $X$ indexed by $[0,1]$ for example. Let $\mathcal{F}(X)=\left(\mathcal{F}_{t}(X), t \in[0,1]\right)$ denote its filtration. The question is under which conditions can we represent any martingale adapted to the filtration of $X$ as a stochastic integral w.r.t. a fixed martingale of the filtration $\mathcal{F}(X)$ ? For the case of Wiener process, this question has a very long history and it is almost impossible to give an exact account of the contributing works. To our knowledge, it has been answered for the first time in the work of K. Itô, cf. [10,15]. In [2], C. Dellacherie has given a different point of view to prove the representation theorem for the Wiener and Poisson processes, based on the uniqueness of their laws. The case of nondegenerate diffusion processes has been elucidated in [12], also studied in [3,11] (cf. also the references there) with also some remarks about the degenerate case. The general case, using the notion of multiplicity is given in [1].

Although the nondegenerate case is completely settled, the degenerate case has not been solved in a definitive way, in the sense that a unique minimal martingale with respect to which the above mentioned property of representation holds, has not been discovered. In this work we are doing exactly this: we prove that for a degenerate diffusion whose law is unique, there exists a minimal martingale with respect to which every square integrable, $\mathcal{F}_{1}(X)$-measurable functional can be represented as a stochastic integral of an $\mathcal{F}(X)$-adapted process. To do this we first prove the density of a class of $\mathcal{F}_{1}(X)$-measurable stochastic integrals in $L^{2}\left(\mathcal{F}_{1}(X)\right)$ using the method launched by C. Dellacherie, then we show that a sequence from this class which is approximating any element of $L^{2}\left(\mathcal{F}_{1}(X)\right)$ defines an $L^{2}$-converging sequence of processes with values in the range of the adjoint of the diffusion coefficient, i.e., with values in $\sigma^{\star}(t, X)\left(\mathbb{R}^{n}\right)$. Because of the degeneracy of $\sigma$, we can not determine the limit of this sequence but its image under the orthogonal projection $P_{t}(X): \mathbb{R}^{d} \rightarrow \sigma^{\star}(t, X)\left(\mathbb{R}^{n}\right)$ and its limit is perfectly well-defined and in this way we see that the minimal martingale for the representation of the functionals of the diffusion (Itô process) is nothing but $d m_{t}=P_{t}(X) d B_{s}$ in its infinitesimal Itô form, where $B$ is the Brownian motion governing the process $X$. 
Note that even the adaptability of $\left(m_{t}, t \in[0,1]\right)$ to the filtration $\mathcal{F}(X)$ is not evident. This result of representation gives also existence of non-orthogonal chaos representation of the elements of $L^{2}\left(\mathcal{F}_{1}(X)\right)$ as the multiple ordered integrals with respect to the martingale $\left(m_{t}, t \in[0,1]\right)$ of elements of $\left\{L^{2}\left(C_{n}, d t_{1} \times \cdots \times d t_{n}\right), n \geq 1\right\}$, where $C_{n}=\left\{\left(t_{1}, \ldots, t_{n}\right) \in[0,1]^{n}: t_{1}>\ldots>t_{n}\right\}$.

In the third section we define the notion of innovation process associated to a degenerate diffusion process ${ }^{1}$. In this case the definition of innovation process is different from the classical case of the perturbation of identity since we have to take into account also the action of the projection operator-valued process $\left(P_{t}(X), t \in[0,1]\right)$. In Section 3 we extend the innovation representation theorem of Fujisaki-Kallianpur-Kunita, [8], under the hypothesis of strong existence and uniqueness to the case of degenerate diffusions. This result confirms the validity of the choice that we have done to define the innovation process. In particular, using this innovation process we can calculate the conditional expectation of the Girsanov exponential of an adapted drift $u$ with respect to the sigma algebra $\mathcal{F}_{1}\left(X^{U}\right)$, where $X^{U}$ is the solution of the diffusion stochastic differential equation where the random input is equal to $U=B+u$.

The results of the third section is then applied to the solution of the adapted MongeAmpère equation in the case of the degenerate diffusions, which extends the results of $[7,13,14]$, cf. also [4-6]. In particular we calculate the relative entropy of the law of $X^{U}$ with respect to the law of $X$ by the use of preceding results.

Let us note to finish this introduction that most of these results are easily extendible to more general situations, for example the strong existence and uniqueness hypothesis can be weakened in the entropic calculations, we have tried to follow maximum homogeneity in the hypothesis and these possible extensions may be treated in separate works.

\section{Stochastic integral representation of functionals of diffusions}

Let $X=\left(X_{t}, t \in[0,1]\right)$ be a weak solution of the following stochastic differential equation:

$$
d X_{t}=b(t, X) d t+\sigma(t, X) d B_{t}, X_{0}=x
$$

where $B=\left(B_{t}, t \in[0,1]\right)$ is an $\mathbb{R}^{d}$-valued Brownian motion and $\sigma:[0,1] \times$ $C\left([0,1], \mathbb{R}^{n}\right) \rightarrow L\left(\mathbb{R}^{d}, \mathbb{R}^{n}\right)$ and $b:[0,1] \times C\left([0,1], \mathbb{R}^{n}\right) \rightarrow \mathbb{R}^{n}$ are measurable maps, adapted to the natural filtration of $C\left([0,1], \mathbb{R}^{n}\right)$ and of linear growth. Recall that the classical theorem of Yamada-Watanabe says that the strong uniqueness implies the uniqueness in law of the above SDE, cf. [16,9]. Hence weak uniqueness is easier to obtain in the applications. In this section we shall assume the weak uniqueness of the equation and prove the martingale representation property for the case where $\sigma$ may be degenerate.

\footnotetext{
1 Note that we use the word diffusion in the large sense, i.e., without demanding a Markov property.
} 
More precisely, let $\left(\mathcal{F}_{t}(X), t \in[0,1]\right)$ be the filtration of $X$ and let us denote by $K$ the set of $\mathbb{R}^{n}$-valued, $\left(\mathcal{F}_{t}(X), t \in[0,1]\right)$-adapted processes $\alpha(X)$, s.t.

$$
E \int_{0}^{1}\left(a(s, X) \alpha_{s}(X), \alpha_{s}(X)\right) d s<\infty
$$

where $a(s, w)=\sigma(s, w) \sigma^{\star}(s, w), s \in[0,1], w \in C\left([0,1], \mathbb{R}^{n}\right)$.

Theorem 1. The set $\Gamma=\left\{N \in L^{2}\left(\mathcal{F}_{1}(X)\right): N=E[N]+\int_{0}^{1}\left(\alpha_{s}(X), \sigma(s, X) d B_{s}\right), \alpha \in K\right\}$ is dense in $L^{2}\left(\mathcal{F}_{1}(X)\right)$.

Proof. Suppose that there is some $M \in L^{2}\left(\mathcal{F}_{1}(X)\right)$ which is orthogonal (in $\left.L^{2}\right)$ to $\Gamma$. Using the usual stopping technique, we can assume that the corresponding $\left(\mathcal{F}_{t}(X), t \in\right.$ $[0,1])$-martingale is bounded and positive whose expectation is equal to one. The orthogonality implies that $\left(M_{t}\left(f\left(X_{t}\right)-\int_{0}^{t} L f(s, X) d s\right), t \in[0,1]\right)$ is again a (local) martingale for any smooth function $f: \mathbb{R}^{n} \rightarrow \mathbb{R}$, where $L$ is defined as

$$
L f(t, X)=\frac{1}{2} \sum_{i, j} a_{i, j}(t, X) \partial_{i, j} f\left(X_{t}\right)+\sum_{i} b_{i}(t, X) \partial_{i} f\left(X_{t}\right)
$$

This implies, with Proposition IV.2.1 of [9], that, under the measure $M \cdot P, X$ is again a weak solution of 2.2. By the uniqueness in law, we get $X(M \cdot P)=X(P)$, since $M$ is $\mathcal{F}_{1}(X)$-measurable, we should have $M=1$. Hence the functionals of the diffusion which are orthogonal to the above set of stochastic integrals are almost surely constant. Consequently, the set $\Gamma$ is total in $L^{2}\left(\mathcal{F}_{1}(X)\right)$.

The following is the extension of the martingale representation theorem to the functionals of degenerate diffusions:

Theorem 2. Denote by $P_{s}(X)$ a measurable version of the orthogonal projection from $\mathbb{R}^{d}$ onto $\sigma(s, X)^{\star}\left(\mathbb{R}^{n}\right) \subset \mathbb{R}^{d}$ and let $F \in L^{2}\left(\mathcal{F}_{1}(X)\right)$ be any random variable with zero expectation. Then there exists a process $\xi(X) \in L_{a}^{2}\left(d t \times d P ; \mathbb{R}^{d}\right)$, adapted to $\left(\mathcal{F}_{t}(X), t \in\right.$ $[0,1])$, such that

$$
F(X)=\int_{0}^{1}\left(P_{s}(X) \xi_{s}(X), d B_{s}\right)_{\mathbb{R}^{d}}=\int_{0}^{1}\left(\xi_{s}(X), P_{s}(X) d B_{s}\right)_{\mathbb{R}^{n}}
$$

a.s.

Conversely, any stochastic integral of the form

$$
\int_{0}^{1}\left(P_{s}(X) \xi_{s}(X), d B_{s}\right)
$$


where $\xi(X)$ is an $\left(\mathcal{F}_{t}(X), t \in[0,1]\right)$-adapted, measurable process with $E \int_{0}^{1}\left|P_{s}(X) \xi_{s}(X)\right|^{2} d s<\infty$, gives rise to an $\mathcal{F}_{1}(X)$-measurable random variable.

Proof. From Theorem 1 , there exists a sequence $\left(F_{n}(X), n \geq 1\right) \subset \Gamma$, where $\Gamma$ is defined in the statement of Theorem 1, converging to $F(X)$ in $L^{2}$. We can suppose that $E\left[F_{n}(X)\right]=0$, for any $n \geq 1$. Hence

$$
F_{n}(X)=\int_{0}^{1}\left(\gamma_{s}^{n}(X), \sigma(s, X) d B_{s}\right)_{\mathbb{R}^{d}}=\int_{0}^{1}\left(\sigma^{\star}(s, X) \gamma_{s}^{n}(X), d B_{s}\right)_{\mathbb{R}^{d}}
$$

As explained above $\gamma^{n}$ is an $\left(\mathcal{F}_{t}(X), t \in[0,1]\right)$-adapted, $\mathbb{R}^{n}$-valued process satisfying $E \int_{0}^{1}\left(a\left(X_{s}\right) \gamma_{s}^{n}(X), \gamma_{s}^{n}(X)\right) d s<\infty$. Since $F_{n}(X) \rightarrow F(X)$ in $L^{2}$,

$$
\lim _{n, m \rightarrow \infty} E \int_{0}^{1}\left|\sigma^{\star}(s, X) \gamma_{s}^{n}(X)-\sigma^{\star}(s, X) \gamma_{s}^{m}(X)\right|^{2} d s=0 .
$$

Let $\alpha_{s}^{n}(X)=\sigma^{\star}\left(X_{s}\right) \gamma_{s}^{n}(X)$, as $P_{s}(X) \alpha_{s}^{n}(S)=P_{s}(X) \sigma^{\star}\left(X_{s}\right) \gamma_{s}^{n}(X)=\sigma^{\star}\left(X_{s}\right) \gamma_{s}^{n}(X)$, $\left(P_{s}(X) \alpha_{s}^{n}(X), n \geq 1\right)$ converges to some $\xi_{s}(X)$ in $L_{a}^{2}\left(d s \times d P ; \mathbb{R}^{d}\right)$. As $P_{s}(X)$ is an orthogonal projection, $\left(P_{s}(X) \alpha_{s}^{n}(X), n \geq 1\right)$ converges to $P_{s}(X) \xi_{s}(X)$ also in $L_{a}^{2}(d s \times$ $\left.d P ; \mathbb{R}^{d}\right)$. Therefore

$$
\begin{aligned}
\int_{0}^{1} P_{s}(X) \xi_{s}(X) \cdot d B_{s} & =\lim _{n} \int_{0}^{1} P_{s}(X) \alpha_{s}^{n}(X) \cdot d B_{s} \\
& =\lim _{n} \int_{0}^{1}\left(\gamma_{s}^{n}(X), \sigma(s, X) d B_{s}\right) \\
& =\lim _{n} F_{n}(X)=F(X) .
\end{aligned}
$$

Let now $G \in L^{2}(P)$ be given by $G=\int_{0}^{1}\left(P_{s}(X) \eta_{s}(X), d B_{s}\right)$ and assume that it is not $\mathcal{F}_{1}(X)$-measurable. Then $G-E\left[G \mid \mathcal{F}_{1}(X)\right]$ is orthogonal to $L^{2}\left(\mathcal{F}_{1}(X)\right)$. It follows from the first part of the theorem that we can represent $E\left[G \mid \mathcal{F}_{1}(X)\right]$ as $\int_{0}^{1}\left(P_{s}(X) \xi_{s}(X), d B_{s}\right)$. Let us define $h=\eta-\xi$, then the orthogonality mentioned above implies that

$$
E\left[\int_{0}^{1}\left(P_{s}(X) h_{s}, d B_{s}\right) \cdot \int_{0}^{1}\left(\alpha_{s}(X), \sigma(s, X) d B_{s}\right)\right]=0
$$

for any $\left(\mathcal{F}_{t}(X), t \in[0,1]\right)$-adapted, measurable $\alpha$ such that $E \int_{0}^{1}\left(a(s, X) \alpha_{s}, \alpha_{s}\right) d s<\infty$. Consequently $P_{s}(X) h_{s}=0 d s \times d P$-a.s., hence $G=E\left[G \mid \mathcal{F}_{1}(X)\right]$ P-a.s. 
Remark. Let $\eta$ be an adapted process such that $\eta_{s}$ belongs to the orthogonal complement of $\sigma^{\star}\left(\mathbb{R}^{n}\right)$ in $\mathbb{R}^{d} d s \times d P$-a.s. Then $\eta+\xi$ can also be used to represent $F(X)$. Hence $\xi(X)$ is not unique but $P(X) \xi(X)$ is always unique.

Theorem 3. Let $\dot{u} \in L^{2}\left(d t \times d P, \mathbb{R}^{d}\right)$ be adapted to the Brownian filtration, then we have

$$
E\left[\int_{0}^{1}\left(\dot{u}_{s}, d B_{s}\right) \mid \mathcal{F}_{1}(X)\right]=\int_{0}^{1}\left(E\left[P_{s}(X) \dot{u}_{s} \mid \mathcal{F}_{s}(X)\right], d B_{s}\right)
$$

almost surely.

Proof. We have to prove first that the right hand side of $(2.4)$ is $\mathcal{F}_{1}(X)$-measurable. We know from Theorem 2 that the left side of (2.4) can be represented as

$$
E\left[\int_{0}^{1}\left(\dot{u}_{s}, d B_{s}\right) \mid \mathcal{F}_{1}(X)\right]=\int_{0}^{1} P_{s}(X) \xi_{s}(X) \cdot d B_{s}
$$

for some $\xi \in L_{a}^{2}\left(d t \times d P ; \mathbb{R}^{d}\right)$. Let $F(X)=\int_{0}^{1} P_{s}(X) \alpha_{s}(X) . d B_{s}$ be any element of $L^{2}\left(\mathcal{F}_{1}(X)\right)$ with $\alpha(X) \in L_{a}^{2}\left(d t \times d P ; \mathbb{R}^{d}\right)$. We have

$$
\begin{aligned}
E\left[\left(\int_{0}^{1} \dot{u}_{s} \cdot d B_{s}\right) F(X)\right] & =E \int_{0}^{1}\left(\dot{u}_{s}, P_{s}(X) \alpha_{s}(X)\right) d s \\
& =E \int_{0}^{1}\left(P_{s}(X) E\left[\dot{u}_{s} \mid \mathcal{F}_{s}(X)\right], P_{s}(X) \alpha_{s}(X)\right) d s \\
& =E\left[\left(\int_{0}^{1} P_{s}(X) \xi_{s}(X) \cdot d B_{s}\right) F(X)\right] \\
& =E \int_{0}^{1}\left(P_{s}(X) \xi_{s}(X), P_{s}(X) \alpha_{s}(X)\right) d s
\end{aligned}
$$

hence $P_{s}(X) \xi_{s}(X)=P_{s}(X) E\left[\dot{u}_{s} \mid \mathcal{F}_{s}(X)\right] d s \times d P$-a.s., in particular

$$
\int_{0}^{1}\left(E\left[P_{s}(X) \dot{u}_{s} \mid \mathcal{F}_{s}(X)\right], d B_{s}\right)
$$

is $\mathcal{F}_{1}(X)$-measurable. The above identification assures then the validity of the relation (2.4). 
Corollary 1. Let $h \in H^{1}\left([0,1], \mathbb{R}^{d}\right)$ (i.e., the Cameron-Martin space), denote by $\rho(\delta h)$ the Wick exponential $\exp \left(\int_{0}^{1}\left(\dot{h}_{s}, d B_{s}\right)-\frac{1}{2} \int_{0}^{1}\left|\dot{h}_{s}\right|^{2} d s\right)$, then we have

$$
E\left[\rho(\delta h) \mid \mathcal{F}_{1}(X)\right]=\exp \left(\int_{0}^{1}\left(P_{s}(X) \dot{h}_{s}, d B_{s}\right)-\frac{1}{2} \int_{0}^{1}\left|P_{s}(X) \dot{h}_{s}\right|^{2} d s\right) .
$$

\section{Theorem 4.}

- Assume that $\mathcal{F}_{t}(X) \subset \mathcal{F}_{t}(B)$ for any $t \in[0,1]$, where $\left(\mathcal{F}_{t}(B), t \in[0,1]\right)$ represents the filtration of the Brownian motion. Define the martingale $m=\left(m_{t}, t \in[0,1]\right)$ as $m_{t}=\int_{0}^{t} P_{s}(X) d B_{s}$, then the set

$$
K=\left\{\rho\left(\delta_{m}(h)\right): h \in H\right\}
$$

is total in $L^{2}\left(\mathcal{F}_{1}(X)\right)$, where $\rho\left(\delta_{m}(h)\right)=\exp \left(\int_{0}^{1}\left(\dot{h}_{s}, d m_{s}\right)-\frac{1}{2} \int_{0}^{1}\left|P_{s}(X) \dot{h}_{s}\right|^{2} d s\right)$. In particular, any element $F$ of $L^{2}\left(\mathcal{F}_{1}(X)\right)$ can be written in a unique way as the sum

$$
F=E[F]+\sum_{n=1}^{\infty} \int_{C_{n}}\left(f_{n}\left(s_{1}, \ldots, s_{n}\right), d m_{s_{1}} \otimes \ldots \otimes d m_{s_{n}}\right)
$$

where $C_{n}$ is the $n$-dimensional simplex in $[0,1]^{n}$ and $f_{n} \in L^{2}\left(C_{n}, d s^{\otimes n}\right) \otimes\left(\mathbb{R}^{d}\right)^{\otimes n}$.

- More generally, without the hypothesis $\mathcal{F}_{t}(X) \subset \mathcal{F}_{t}(B)$, for any $F \in L^{2}\left(\mathcal{F}_{1}(X)\right) \cap$ $L^{2}\left(\mathcal{F}_{1}(B)\right)$, the conclusions of the first part of the theorem hold true.

Proof. Let $F \in L^{2}\left(\mathcal{F}_{1}(X)\right)$, assume that $F$ is orthogonal to $K$, i.e. $E\left[F \rho\left(\delta_{m}(h)\right)\right]=0$ for any $h \in H$. From Corollary $1 \rho\left(\delta_{m}(h)\right)=E\left[\rho(\delta h) \mid \mathcal{F}_{1}(X)\right]$, hence

$$
E[F \rho(\delta h)]=E\left[F \rho\left(\delta_{m}(h)\right)\right]=0,
$$

hence $F$ is also orthogonal to $\mathcal{E}=\{\rho(\delta h): h \in H\}$, which is total in $L^{2}\left(\mathcal{F}_{1}(B)\right.$, where $\mathcal{F}_{1}(B)$ is the $\sigma$-algebra generated by the governing Brownian motion, therefore $F=0$. Consequently the span of $K$ is dense in $L^{2}\left(\mathcal{F}_{1}(X)\right)$. Theorem 3 and Corollary 1 allow us to calculate the conditional expectations of the multiple Wiener integrals w.r.t. $\mathcal{F}_{1}(X)$ and the result will be multiple iterated stochastic integrals w.r.t. $m$ in the form given by the formula (2.5); here we have to be careful as the operator valued process $\left(P_{s}(X), s \in[0,1]\right)$ is not deterministic, the symmetric interpretation of the Ito-Wiener integrals w.r.t. the Brownian motion is no longer valid in our case and they have to be written as iterated Ito integrals.

Remark 1. In this theorem we need to make the hypothesis $\mathcal{F}_{t}(X) \subset \mathcal{F}_{t}(B)$ for any $t \in$ $[0,1]$ to assure the non-symmetric chaos representation $(2.5)$. Without this hypothesis, 
although we have Theorem 2 and when we iterate it we have a similar representation, but, consisting of a finite number of terms. It is not possible to push this procedure up to infinity since we have no control at infinity.

Remark 2. Let us note that if there is no strong solution to the equation defining the process $X$, the chaotic representation property may fail. For example, let $U$ be a weak solution of

$$
d U_{t}=\alpha_{t}(U) d t+d B_{t}
$$

with $U_{0}$ given. Assume that (2.6) has no strong solution, as it may happen in the famous example of Tsirelson ([9]), i.e., $U$ is not measurable w.r.t. the sigma algebra generated by $B$, then we have no chaotic representation property for the elements of $L^{2}\left(\mathcal{F}_{1}(U)\right)$ in terms of the iterated stochastic integrals of deterministic functions on $C_{n}, n \in \mathbb{N}$ w.r.t. $B$; the contrary would imply the equality of $\mathcal{F}_{1}(U)$ and of $\mathcal{F}_{1}(B)$, which would contradict the non-existence of strong solutions.

\section{Innovation process}

In this section we assume that the SDE 2.2 has unique strong solution. To fix the ideas, we can suppose that $\sigma$ and $b$ satisfy the following kind of Lipschitz condition on the path space: for $\xi, \eta \in C\left([0,1], \mathbb{R}^{n}\right)$ :

$$
|\gamma(t, \xi)-\gamma(t, \eta)| \leq K \sup _{s \leq t}|\xi(s)-\eta(s)|
$$

for $\gamma$ being equal either to $\sigma$ or to $b$ with corresponding Euclidean norms at the left hand side.

Assume that $\dot{u} \in L^{2}\left(d t \times d P, \mathbb{R}^{d}\right)$ is a process adapted to the filtration of Brownian motion, let $U=\left(U_{t}, t \in[0,1]\right)$ be defined as $U_{t}=B_{t}+\int_{0}^{t} \dot{u}_{s} d s$. We denote by $X^{U}$ the strong solution of the equation

$$
\begin{aligned}
d X_{t}^{U} & =\sigma\left(t, X^{U}\right) d U_{t}+b\left(t, X^{U}\right) d t \\
& =\sigma\left(t, X^{U}\right)\left(d B_{t}+\dot{u}_{t} d t\right)+b\left(t, X^{U}\right) d t
\end{aligned}
$$

Since $B$ is the canonical Brownian motion, we have $X_{t}^{U}=X_{t} \circ U$ a.s. Moreover, if $\eta \in \mathbb{R}^{n}$ is any vector, we have

$$
\begin{aligned}
\left(\sigma\left(t, X^{U}\right) \dot{u}_{t}, \eta\right) & =\left(\dot{u}_{t}, \sigma\left(t, X^{U}\right)^{\star} \eta\right) \\
& =\left(P_{t}\left(X^{U}\right) \dot{u}_{t}, \sigma\left(t, X^{U}\right)^{\star} \eta\right) \\
& =\left(\sigma\left(t, X^{U}\right) P_{t}\left(X^{U}\right) \dot{u}_{t}, \eta\right),
\end{aligned}
$$


where $P_{t}\left(X^{U}\right)$ denotes the orthogonal projection from $\mathbb{R}^{d}$ onto $\sigma\left(t, X^{U}\right)^{\star}\left(\mathbb{R}^{n}\right)$. Hence, in order to define a reasonably useful concept of innovation, we should estimate the perturbation $\dot{u}$ simultaneously w.r.t. the both projections, i.e., with respect to the conditional expectation $E\left[\cdot \mid \mathcal{F}_{s}\left(X^{U}\right)\right]$ (which is a projection) and also w.r.t. $P_{s}\left(X^{U}\right)$ : Let $Z=\left(Z_{t}, t \in[0,1]\right.$ ) (to avoid the ambiguity we shall also use the notation $Z^{U}$ if necessary) be defined as

$$
Z_{t}=B_{t}+\int_{0}^{t}\left(\dot{u}_{s}-E\left[P_{s}\left(X^{U}\right) \dot{u}_{s} \mid \mathcal{F}_{s}\left(X^{U}\right)\right]\right) d s
$$

where $\left(\mathcal{F}_{s}\left(X^{U}\right), s \in[0,1]\right)$ denotes the filtration of $X^{U}$.

Proposition 1. The process $\left(\int_{0}^{t} P_{s}\left(X^{U}\right) d Z_{s}, t \in[0,1]\right)$ is an $\left(\mathcal{F}_{t}\left(X^{U}\right), t \in[0,1]\right)$-local martingale.

Proof. Assume to begin that $|u|_{H}^{2}=\int_{0}^{1}\left|\dot{u}_{s}\right|^{2} d s \in L^{\infty}(P)$. Let us first prove that the process under question is adapted to the filtration $\left(\mathcal{F}_{t}\left(X^{U}\right), t \in[0,1]\right)$ : From the Girsanov theorem, the process $\left(\int_{0}^{t} \sigma\left(s, X^{U}\right) d U_{s}, t \in[0,1]\right)$ is adapted to the filtration $\left(\mathcal{F}_{t}\left(X^{U}\right), t \in\right.$ $[0,1])$, using the same method as in Theorem 2 by replacing $B$ by $U$ and the probability $d P$ by $\rho(-\delta u) d P$, we conclude that the process $\left(\int_{0}^{t} P_{s}\left(X^{U}\right) d U_{s}, t \in[0,1]\right)$, and hence the process $\left(\int_{0}^{t} P_{s}\left(X^{U}\right) d Z_{s}, t \in[0,1]\right)$ is adapted to the filtration $\left(\mathcal{F}_{t}\left(X^{U}\right), t \in[0,1]\right)$. To show the (local) martingale property it suffices to write that

$$
\int_{0}^{t} P_{s}\left(X^{U}\right) d Z_{s}=\int_{0}^{t} P_{s}\left(X^{U}\right) d B_{s}+\int_{0}^{t} P_{s}\left(X^{U}\right)\left[\dot{u}_{s}-E\left[\dot{u}_{s} \mid \mathcal{F}_{s}\left(X^{U}\right)\right]\right] d s
$$

from which the martingale property follows. The general case follows from a stopping argument.

Remark. Note that $Z=Z^{U}$ is not a Brownian motion.

Theorem 5. Let $\dot{u} \in L^{2}\left(d t \times d P, \mathbb{R}^{d}\right)$ be such that $E[\rho(-\delta u)]=1$, then we have

$$
\begin{aligned}
\zeta_{t} & =E\left[\rho(-\delta u) \mid \mathcal{F}_{t}\left(X^{U}\right)\right] \\
& =\exp \left(-\int_{0}^{t} P_{s}\left(X^{U}\right) E\left[\dot{u}_{s} \mid \mathcal{F}_{s}\left(X^{U}\right)\right] \cdot d Z_{s}-\frac{1}{2} \int_{0}^{t}\left|P_{s}\left(X^{U}\right) E\left[\dot{u}_{s} \mid \mathcal{F}_{s}\left(X^{U}\right)\right]\right|^{2} d s\right) .
\end{aligned}
$$

Proof. Assume first that $|u|_{H}^{2}=\int_{0}^{1}\left|\dot{u}_{s}\right|^{2} d s \in L^{\infty}(P)$. Let $\left(X_{t}^{U}, t \in[0,1]\right)$ be the (strong) solution of $d X_{t}=\sigma\left(X_{t}\right) d U_{t}$, where $d U_{t}=d B_{t}+\dot{u}_{t} d t$ and let $f$ be a $C^{2}$-function on $\mathbb{R}^{n}$. Using the Itô formula, we calculate the Doob-Meyer process associated to the semimartingales $\left(\zeta_{t}\right)$ and $\left(f\left(X_{t}^{U}\right)\right)$ : 


$$
\left\langle f \circ X^{U}, \zeta\right\rangle_{t}=-\int_{0}^{t}\left(D f\left(X_{s}^{U}\right), \sigma\left(s, X^{U}\right) P_{s}\left(X^{U}\right) E\left[\dot{u}_{s} \mid \mathcal{F}_{s}\left(X^{U}\right)\right]\right) d s .
$$

Let $f \in C^{2}$, using again the Itô formula and the relation (3.8) we get

$$
\begin{aligned}
f\left(X_{t}^{U}\right) \zeta_{t}= & \int_{0}^{t} f\left(X_{s}^{U}\right) d \zeta_{s}+\int_{0}^{t} \zeta_{s}(L f)\left(s, X^{U}\right) d s+\int_{0}^{t} \zeta_{s}\left(D f\left(X_{s}^{U}\right), \sigma\left(s, X^{U}\right) d U_{s}\right) \\
& -\int_{0}^{t}\left(D f\left(X_{s}^{U}\right), \sigma\left(s, X^{U}\right) P_{s}\left(X^{U}\right) E\left[\dot{u}_{s} \mid \mathcal{F}_{s}\left(X^{U}\right)\right]\right) d s
\end{aligned}
$$

where $L f$ is defined by the relation 2.3. Therefore the process

$$
\left.\left(f\left(X_{t}^{U}\right) \zeta_{t}-\int_{0}^{t}(L f)\left(s, X^{U}\right)\right) \zeta_{s} d s, t \in[0,1]\right)
$$

is a $P$-local martingale, therefore, by the uniqueness in law of the solution, we should have

$$
E\left[\zeta_{1} F\left(X^{U}\right)=E\left[F(X)=E\left[F\left(X^{U}\right) \rho(-\delta u)\right]\right.\right.
$$

for any $F \in C_{b}$ on the path space, and the last inequality follows from the Girsanov theorem. It suffices then to remark that $\zeta_{1}$ is $\mathcal{F}_{1}\left(X^{U}\right)$-measurable by Theorem 3 and again by the Girsanov theorem which allows us to replace $B$ by $U$. The general case follows from the usual stopping time argument: let $T_{n}=\inf \left(t: \int_{0}^{t}\left|\dot{u}_{s}\right|^{2} d s \geq n\right)$ and define $\dot{u}_{s}^{n}=1_{\left[0, T_{n}\right]}(s) \dot{u}_{s}$ and let $U^{n}=B+\int_{0}^{\dot{u}} \dot{u}_{s}^{n} d s$. Then $X^{U^{n}}$ converges almost surely uniformly to $X^{U}$, hence $\lim _{n} E\left[\cdot \mid \mathcal{F}_{t}\left(X^{U^{n}}\right)\right]=E\left[\cdot \mid \mathcal{F}_{t}\left(X^{U}\right)\right] d t$-almost surely as bounded operators on $L^{1}(P)$ and $\left(P_{s}\left(X^{U^{n}}\right), n \geq 1\right)$ converges to $P_{s}(X) d s \times d P$-almost surely. Moreover $\left(\rho\left(-\delta u^{n}\right), n \geq 1\right)$ converges strongly in $L^{1}(P)$ to $\rho(-\delta u)$, therefore the general case follows.

The following result is the generalization of the celebrated innovation's theorem to the degenerate case, cf. [8]:

Theorem 6. Let $\left(M_{t}, t \in[0,1]\right)$ be a square integrable $\left(P,\left(\mathcal{F}_{t}\left(X^{U}\right), t \in[0,1]\right)\right)$-martingale, then it can be represented as a stochastic integral of an $\left(\mathcal{F}_{t}\left(X^{U}\right), t \in[0,1]\right)$-adapted, $\mathbb{R}^{d}$-valued process $\beta\left(X^{U}\right)$ in the following way:

$$
M_{t}=M_{0}+\int_{0}^{t}\left(P_{s}\left(X^{U}\right) \beta_{s}\left(X^{U}\right), d Z_{s}\right)
$$


P-a.s., where

$$
\int_{0}^{t}\left|P_{s}\left(X^{U}\right) \beta_{s}\left(X^{U}\right)\right|^{2} d s<\infty
$$

P-a.s., for any $t \in[0,1]$.

Proof. Assume that $M$ is a $\left(P,\left(\mathcal{F}_{t}\left(X^{U}\right), t \in[0,1]\right)\right)$-martingale, then for any $s<t$ and $A \in \mathcal{F}_{s}\left(X^{U}\right)$ we have

$$
\begin{aligned}
E\left[\frac{M_{t}}{\zeta_{t}} 1_{A} \rho(-\delta u)\right] & =E\left[\frac{M_{t}}{\zeta_{t}} 1_{A} \zeta_{t}\right] \\
& =E\left[M_{t} 1_{A}\right]=E\left[M_{s} 1_{A}\right] \\
& =E\left[\frac{M_{s}}{\zeta_{s}} 1_{A} \rho(-\delta u)\right]
\end{aligned}
$$

where $\zeta$ is the optional projection of $\rho(-\delta u)$ w.r.t. the filtration $\left(\mathcal{F}_{t}\left(X^{U}\right), t \in[0,1]\right)$ as calculated in Theorem 5. Consequently $\left(M_{t} / \zeta_{t}, t \in[0,1]\right)$ is a $\left(Q,\left(\mathcal{F}_{t}\left(X^{U}\right), t \in\right.\right.$ $[0,1]))$-martingale, where $d Q=\rho(-\delta u) d P$. As $U$ is a $Q$-Brownian motion, from Theorem 2 , we can represent $\left(M_{t} / \zeta_{t}, t \in[0,1]\right)$ as

$$
\frac{M_{t}}{\zeta_{t}}=c+\int_{0}^{t}\left(P_{s}\left(X^{U}\right) \dot{\alpha}_{s}\left(X^{U}\right), d U_{s}\right)
$$

then using the Itô formula

$$
\begin{aligned}
M_{t}= & \frac{M_{t}}{\zeta_{t}} \zeta_{t} \\
= & c+\int_{0}^{t} \zeta_{s}\left(P_{s}\left(X^{U}\right) \dot{\alpha}_{s}\left(X^{U}\right), d U_{s}\right)-\int_{0}^{t} \frac{M_{s}}{\zeta_{s}} \zeta_{s}\left(P_{s}\left(X^{U}\right) E\left[\dot{u}_{s} \mid \mathcal{F}_{s}\left(X^{U}\right)\right], d Z_{s}\right) \\
& -\int_{0}^{t} \zeta_{s}\left(P_{s}\left(X^{U}\right) \dot{\alpha}_{s}\left(X^{U}\right), E\left[\dot{u}_{s} \mid \mathcal{F}_{s}\left(X^{U}\right)\right]\right) d s \\
= & c+\int_{0}^{t} \zeta_{s}\left(P_{s}\left(X^{U}\right) \dot{\alpha}_{s}\left(X^{U}\right), d Z_{s}+P_{s}\left(X^{U}\right) E\left[\dot{u}_{s} \mid \mathcal{F}_{s}\left(X^{U}\right)\right]\right) d s \\
& -\int_{0}^{t} M_{s}\left(P_{s}\left(X^{U}\right) E\left[\dot{u}_{s} \mid \mathcal{F}_{s}\left(X^{U}\right)\right], d Z_{s}\right)
\end{aligned}
$$




$$
\begin{aligned}
& -\int_{0}^{t} \zeta_{s}\left(P_{s}\left(X^{U}\right) \dot{\alpha}_{s}\left(X^{U}\right), E\left[\dot{u}_{s} \mid \mathcal{F}_{s}\left(X^{U}\right)\right]\right) d s \\
= & c+\int_{0}^{t}\left(P_{s}\left(X^{U}\right)\left[\zeta_{s} \dot{\alpha}_{s}\left(X^{U}\right)-M_{s} E\left[\dot{u}_{s} \mid \mathcal{F}_{s}\left(X^{U}\right)\right]\right], d Z_{s}\right)
\end{aligned}
$$

and this completes the proof.

\section{Entropy calculation and Monge-Ampère equation}

Assume that $l(X)$ is a probability density measurable w.r.t. $\mathcal{F}_{1}(X)$, i.e., $E[l(X)]=1$ and with finite entropy: $E[l(X) \log l(X)]<\infty$. We want to find a process $U=B+u=$ $B+\int_{0}^{\cdot} \dot{u}_{s} d s$ which is an adapted perturbation of the Brownian motion $B$ such that

$$
l(X)=\frac{d X^{U}(P)}{d X(P)} \circ X
$$

This problem is called the causal Monge-Ampère problem. To simplify the calculations, we shall assume that $l \circ X$ is $P$-a.s. strictly positive. Assume that such a $U$ (hence $u$ ) exists and that $u$ satisfies the Girsanov theorem, i.e., $E\left[\rho\left(-\delta_{B} u\right)\right]=1$. Then the Girsanov theorem implies that

$$
l \circ X^{U} E\left[\rho(-\delta u) \mid \mathcal{F}_{1}\left(X^{U}\right)\right]=1
$$

$P$-a.s., which is the causal version of the Monge-Ampère equation. From Theorem 2, $l \circ X$ can be represented as

$$
l \circ X=\exp \left(-\int_{0}^{1} P_{s}(X) \dot{v}_{s}(X) \cdot d B_{s}-\frac{1}{2} \int_{0}^{1}\left|P_{s}(X) \dot{v}_{s}(X)\right|^{2} d s\right)
$$

where $\left(P_{s}(X) \dot{v}_{s}(X), s \in[0,1]\right)$ is adapted to the filtration of $X$ and $\int_{0}^{1}\left|P_{s}(X) \dot{v}_{s}(X)\right|^{2} d s<\infty P$-a.s. Besides, since $U$ is a Brownian motion under the probability $\rho(-\delta u) d P$, it follows from Theorem 2 that $l \circ X^{U}$ can be represented as

$$
l \circ X^{U}=\exp \left(-\int_{0}^{1} P_{s}\left(X^{U}\right) \dot{v}_{s}\left(X^{U}\right) \cdot d U_{s}-\frac{1}{2} \int_{0}^{1}\left|P_{s}\left(X^{U}\right) \dot{v}_{s}\left(X^{U}\right)\right|^{2} d s\right) .
$$

Inserting the right hand side of (4.10) and $E\left[\rho(-\delta u) \mid \mathcal{F}_{1}\left(X^{U}\right)\right]$ which is already calculated in Theorem 5 in the Monge-Ampère equation (4.9) and then taking the logarithm of the final expression, we obtain 


$$
\begin{aligned}
& \int_{0}^{1} P_{s}\left(X^{U}\right)\left(E\left[\dot{u}_{s} \mid \mathcal{F}_{s}\left(X^{U}\right)\right]+\dot{v}_{s}\left(X^{U}\right)\right) \cdot d Z_{s} \\
& +\frac{1}{2} \int_{0}^{1}\left(\left|P_{s}\left(X^{U}\right) \dot{v}_{s}\left(X^{U}\right)+P_{s}\left(X^{U}\right) E\left[\dot{u}_{s} \mid \mathcal{F}_{s}\left(X^{U}\right)\right]\right|^{2}\right) d s=0 .
\end{aligned}
$$

This relation implies that

$$
P_{s}\left(X_{s}^{U}\right)\left(\dot{v}_{s}\left(X^{U}\right)+E\left[\dot{u}_{s} \mid \mathcal{F}_{s}\left(X^{U}\right)\right]\right)=0
$$

$d s \times d P$-almost surely, which is a quite elaborate nonlinear equation. From the MongeAmpère equation (4.9) we can calculate the relative entropy between $X^{U}(P)$ and $X(P)$, denoted by $H\left(X^{U}(P) \mid X(P)\right)$ :

Theorem 7. Suppose that $l$ is an $X(P)$-almost surely strictly positive density. There exists some $\dot{u} \in L^{2}(d s \times d P)$ with $E[\rho(-\delta u)]=1$ with

$$
\frac{d X^{U}(P)}{d X(P)}=l
$$

if and only if

$$
P_{s}\left(X_{s}^{U}\right)\left(\dot{v}_{s}\left(X^{U}\right)+E\left[\dot{u}_{s} \mid \mathcal{F}_{s}\left(X^{U}\right)\right]\right)=0
$$

In this case we also have

$$
H\left(X^{U}(P) \mid X(P)\right)=\frac{1}{2} E \int_{0}^{1}\left|P_{s}\left(X^{U}\right) E\left[\dot{u}_{s} \mid \mathcal{F}_{s}\left(X^{U}\right)\right]\right|^{2} d s=\frac{1}{2} E \int_{0}^{1}\left|P_{s}\left(X^{U}\right) \dot{v}_{s}\left(X^{U}\right)\right|^{2} d s .
$$

Proof.

$$
\begin{aligned}
H\left(X^{U}(P) \mid X(P)\right) & =\int \log \frac{d X^{U}(P)}{d X(P)} d X^{U}(P) \\
& =\int \log \frac{d X^{U}(P)}{d X(P)} \circ X^{U} d P \\
& =\int \log l \circ X^{U} d P \\
& =\frac{1}{2} E \int_{0}^{1}\left|P_{s}\left(X^{U}\right) E\left[\dot{u}_{s} \mid \mathcal{F}_{s}\left(X^{U}\right)\right]\right|^{2} d s,
\end{aligned}
$$

provided that $\dot{u} \in L^{2}(d s \times d P)$ and the first equality follows, the second one is a consequence of the relation (4.11), it can be also proven directly from the Girsanov theorem. 
Proposition 2. Assume that $l$ and $u$ are given as above. Suppose furthermore that

$$
H\left(X^{U}(P) \mid X(P)\right)=\frac{1}{2} E \int_{0}^{1}\left|P_{s}\left(X^{U}\right) \dot{u}_{s}\right|^{2} d s
$$

Then the following equation holds true:

$$
P_{s}\left(X^{U}\right) d U_{s}+P_{s}\left(X^{U}\right) \dot{v}_{s} \circ X^{U} d s=P_{s}\left(X^{U}\right) d B_{s}
$$

almost surely. In particular, $X^{U}$ satisfies the following stochastic differential equation:

$$
d X_{t}^{U}=\sigma\left(t, X^{U}\right)\left(d B_{t}-\dot{v}_{t} \circ X^{U} d t\right)+b\left(t, X^{U}\right) d t
$$

with the same initial condition as $X$.

Proof. The hypothesis (4.12) implies that the process $\left(P_{t}\left(X^{U}\right) \dot{u}_{t}, t \in[0,1]\right)$ is $d s$-almost surely adapted to the filtration $\left(\mathcal{F}_{t}\left(X^{U}\right), t \in[0,1]\right)$, hence we get from the equality (4.11) the relation

$$
P_{t}\left(X^{U}\right)\left(\dot{v}_{t} \circ X^{U}+\dot{u}_{t}\right)=0
$$

which implies at once the relation (4.13). To see the next one, note that

$$
\begin{aligned}
d X_{t}^{U} & =\sigma\left(t, X^{U}\right)\left(d B_{t}+\dot{u}_{t} d t\right)+b\left(t, X^{U}\right) d t \\
& =\sigma\left(t, X^{U}\right)\left(d B_{t}+P_{t}\left(X^{U}\right) \dot{u}_{t} d t\right)+b\left(t, X^{U}\right) d t \\
& =\sigma\left(t, X^{U}\right)\left(d B_{t}-P_{t}\left(X^{U}\right) \dot{v}_{t} \circ X^{U} d t\right)+b\left(t, X^{U}\right) d t \\
& =\sigma\left(t, X^{U}\right)\left(d B_{t}-\dot{v}_{t} \circ X^{U} d t\right)+b\left(t, X^{U}\right) d t
\end{aligned}
$$

where we have used the fact that $\sigma\left(t, X^{U}\right) \eta=\sigma\left(t, X^{U}\right) P_{t}\left(X^{U}\right) \eta$ for any vector in $\mathbb{R}^{d}$ since $P_{t}\left(X^{U}\right)$ is the orthogonal projection of $\mathbb{R}^{d}$ onto $\sigma\left(X_{t}^{U}\right)^{\star}\left(\mathbb{R}^{n}\right)$.

Theorem 7 can be extended as follows

Theorem 8. Assume that $u \in L_{a}^{2}(d t \times d P, H)$ and denote by $U$ the process $\left(B_{t}+\int_{0}^{t} \dot{u}_{s} d s, t \in\right.$ $[0,1])$. assume also, as before, the Lipschitz hypothesis about the drift and diffusion coefficients, then the following inequality holds true:

$$
H\left(X^{U}(P) \mid X(P)\right) \leq \frac{1}{2} E \int_{0}^{1}\left|P_{s}\left(X^{U}\right) E\left[\dot{u}_{s} \mid \mathcal{F}_{s}\left(X^{U}\right)\right]\right|^{2} d s
$$


Proof. If $u \in L_{a}^{\infty}(d t \times d P, H)$, then the claim with equality (instead of inequality) follows from Theorem 7 . For the case $u \in L_{a}^{2}(d t \times d P, H)$, define $T_{n}=\inf \left(t>0: \int_{0}^{t}\left|\dot{u}_{s}\right|^{2} d s>n\right)$, then $u^{n}$ defined by

$$
u^{n}(t)=\int_{0}^{t} 1_{\left[0, T_{n}\right]}(s) \dot{u}_{s} d s
$$

is in $L_{a}^{\infty}(d t \times d P, H)$, hence we have

$$
H\left(X^{U^{n}}(P) \mid X(P)\right)=\frac{1}{2} E \int_{0}^{1}\left|P_{s}\left(X^{U^{n}}\right) E\left[1_{\left[0, T_{n}\right]}(s) \dot{u}_{s} \mid \mathcal{F}_{s}\left(X^{U^{n}}\right)\right]\right|^{2} d s .
$$

As $n \rightarrow \infty,\left(X^{U^{n}}(P), n \geq 1\right)$ converges weakly to $X^{U}(P)$ and the weak lower semicontinuity of the entropy implies that

$$
\begin{aligned}
H\left(X^{U}(P) \mid X(P)\right) & \leq \liminf _{n} \frac{1}{2} E \int_{0}^{1}\left|P_{s}\left(X^{U^{n}}\right) E\left[1_{\left[0, T_{n}\right]}(s) \dot{u}_{s} \mid \mathcal{F}_{s}\left(X^{U^{n}}\right)\right]\right|^{2} d s \\
& =\frac{1}{2} E \int_{0}^{1}\left|P_{s}\left(X^{U}\right) E\left[\dot{u}_{s} \mid \mathcal{F}_{s}\left(X^{U}\right)\right]\right|^{2} d s,
\end{aligned}
$$

where the limit of the right hand side of the equation (4.16) follows from the Lipschitz hypothesis.

\section{References}

[1] M.H.A. Davis, P. Varaiya, The multiplicity of an increasing family of $\sigma$-fields, Ann. Probab. 2 (1974) 958-963.

[2] C. Dellacherie, Intégrales stochastiques par rapport aux processus de Wiener ou de Poisson, in: Séminaire de Probabilités VIII, in: Lecture Notes in Mathematics, vol. 381, Springer-Verlag, 1973, Correction dans SP IX, in: LNM, vol. 465.

[3] N. El Karoui, H. Reinhard, Processus de diffusion dans $\mathbb{R}^{n}$, in: Séminaire de Probabilités, VII (Univ. Strasbourg, année universitaire 1971-1972), in: Lecture Notes in Math., vol. 321, Springer, Berlin, 1973, pp. 95-117.

[4] D. Feyel, A.S. Üstünel, Transport of measures on Wiener space and the Girsanov theorem, C. R. Math. 334 (1) (2002) 1025-1028.

[5] D. Feyel, A.S. Üstünel, Monge-Kantorovitch measure transportation and Monge-Ampère equation on Wiener space, Probab. Theory Related Fields 128 (3) (2004) 347-385.

[6] D. Feyel, A.S. Üstünel, Monge-Kantorovitch measure transportation, Monge-Ampère equation and the Itô calculus, in: Advanced Studies in Pure Mathematics, Math. Soc. of Japan, vol. 41, Mathematical Society of Japan, 2004, pp. 32-49.

[7] D. Feyel, A.S. Üstünel, M. Zakai, Realization of positive random variables via absolutely continuous transformations of measure on Wiener space, Probab. Surv. 3 (2006) 170-205 (electronic).

[8] M. Fujisaki, G. Kallianpur, H. Kunita, Stochastic differential equations for the nonlinear filtering problem, Osaka J. Math. 9 (1972) 19-40.

[9] N. Ikeda, S. Watanabe, Stochastic Differential Equations and Diffusion Processes, 1981. 
[10] K. Itô, Multiple Wiener integrals, J. Math. Soc. Japan 3 (1951) 157-169.

[11] J. Jacod, Calcul stochastique et problémes de martingales, Lecture Notes in Mathematics, vol. 714, Springer-Verlag, 1979.

[12] D.W. Stroock, S.R.S. Varadhan, Diffusion processes with continuous coefficients 1, Comm. Pure Appl. Math. 22 (1969) 345-400.

[13] A.S. Üstünel, Entropy, invertibility and variational calculus of adapted shifts on Wiener space, J. Funct. Anal. 257 (8) (2009) 3655-3689.

[14] A.S. Üstünel, Variational calculation of Laplace transforms via entropy on Wiener space and applications, J. Funct. Anal. 267 (8) (2014) 3058-3083.

[15] S. Watanabe, The Japanese contributions to martingales, J. Électron. Hist. Probab. Stat. (J. Electron. Hist. Probab. Stat.) 5 (1) (Juin/June 2009).

[16] Y. Yamada, S. Watanabe, On the uniqueness of solutions of stochastic differential equations, J. Math. Kyoto Univ. 11 (1971) 155-167. 